\title{
Eye-hand coordination necessary for skilled movements in calligraphy
}

\author{
Atsuo MURATA ${ }^{1 \dagger}$ \\ ${ }^{1}$ Graduate School of Natural Science and Technology, Okayama University
}

\begin{abstract}
The aim of this study was to explore eye-hand coordination skill necessary for enhancing performance from the perspective of simultaneous measurement of eye-gaze and pen tip locations during a calligraphy task. The developed simultaneous measurement system of pen tip and eye-gaze location succeeded in extracting eye-hand coordination ability necessary for acquiring a higher skill in calligraphy. Experts tended to execute eye movements preceding brush tip. The $x$ - and $y$-directional differences between brush tip and eye-gaze point were larger for experts. Moreover, the fluctuation of pen pressure of experts tended to be larger than that of novices.
\end{abstract}

Key words : eye-hand coordination, eye-gaze, brush tip location, simultaneous measurement of eye-hand coordination, writing (pen) pressure.

\section{Introduction}

Eye-hand coordination, which corresponds to the coordinated control of hand movements with the guidance of eye movements, is necessary for the simultaneous use of our hands and eyes in a variety of activities, such as taking note information written on a white board, calligraphy, driving a car, or batting. Our eyes are used to direct attention to a stimulus or target of our action and help our motor system to act according to our plan and move our hands to simultaneously carry out the planned action ${ }^{1,2)}$. Eye-hand coordination needs a complex cognitive ability to integrate visual and motor system and guide our hand movements according to the visual perception. During the integration process of our visual and motor system, we must use visual information to correct a behavior that isn't appropriate for a situation ${ }^{3,4)}$. Without appropriate eye-hand coordination, it is impossible to carry out even the simplest of actions such as picking up a book from a table or taking note.

The neural control of eye-hand coordination is performed by the visual and perceptual system, the central nervous system, and the motor response (peripheral nervous system). The frontal and parietal cortex areas of our brain are especially related to the control of eye saccades and hand movement, and play a key role in eye-hand coordination and the planning of

2020 年 6 月 15 日受付

${ }^{\dagger}$ 3-1-1, Tsushimanaka, Kita-ku, Okayama city, Okayama, 700-8530

Dept. of Intelligent Mechanical Systems, Graduate School of Natural Science and Technology, Okayama University

Atsuo MURATA

Tel: 086-251-8055

E-mail:murata@iims.sys.okayama-u.ac.jp movements during tasks. In this process, proprioception and transformation of motor sensory input is related to plan and control movement ${ }^{5)}$.

Humans proactively control eye-gaze to guide their movement ${ }^{3,4)}$. The eyes usually fixate on a target before the hands undertake movements. This indicates that the eyes provide the hands with spatial information for movements. The duration for which the eyes are locked onto a target of hand movements varies. The eye-gazes sometimes remain fixated until the movement is completed. At other times, the eyes saccade toward other objects of interest before actual hand movements occur.

An analysis of skill acquisition process is a very important in the field of cognitive and movement sciences ${ }^{6,7)}$. In a variety of tasks, the information obtained by visual information processing is essential for the determination and the execution of physical movements. It is assumed that the relationship between the eye movement and the physical movement differs according to the skill level. In order to learn and master the traditional skills efficiently, the research focusing on the cognitive processes and motor process is necessary and indispensable. Therefore, the exploration of the difference of eye-hand coordination between skilled and non-skilled participants would contribute to the development of effective tradition method of skill.

Although it has been well established that eye-hand coordination is neutrally controlled by the visual and perceptual system, the central nervous system (neocortex), and the motor response (peripheral nervous system), it is necessary to acquire a method or means to measure simultaneously eye and hand movements to get further insights into eye-hand coordination. It is at present to measure eye and hand movements separately. 
However, there are few methods or means to visualize this process of eye-hand coordination.

Vickers $^{6}$ clarified different features of eye-hand coordination between skilled and non-skilled free-throw shooters. The ability of eye-hand coordination and the ability of recognizing and predicting the work area are important elements in skilled tasks ${ }^{7}$. Sano and $\mathrm{Ukida}^{8)}$, applying image processing techniques to the evaluation of characters in calligraphy, showed that the writing of characteristic components of calligraphy differed between skilled and non-skilled participants. The simultaneous measurement technique of eye-gaze and hand location is halfway, and is not systematically described so that a similar measurement system of eye-hand coordination can be made easily and with high measurement accuracy.

Therefore, Murata and Inoue ${ }^{9)}$ developed a system that can simultaneously measure the eye-gaze and the brush tip locations has been developed, and its algorithm has been systematically described. They construct bases for clarifying the process of skill acquisition from the viewpoint of eyehand coordination. First, Murata and Inoue ${ }^{9)}$ engaged in the following development: (1) calculation of pen tip location, (2) calculation of eye-gaze location after correction of binocular parallax, and (3) transformation of eye-gaze location to the same coordination system with pen tip location. They used calligraphy as a demonstrative application of simultaneous measurements of eye-gaze and brush tip location.

This study explored eye-hand coordination skill necessary for enhancing performance from the perspective of simultaneous measurement of eye-gaze and pen tip locations and pen (writing) pressure during a calligraphy task to get further insights into eye-hand coordination necessary for skilled movements.

\section{Extraction of characteristics necessary for skilled calligraphy using simultaneous measurement of eye-gaze and brush tip location in calligraphy}

Murata and Inoue ${ }^{9)}$ mentioned the calculation method of pen tip location, calculation method of eye-gaze location after correction of binocular parallax, and calculation method for eye-hand coordination system that transforms eye-gaze locations after correcting binocular parallax to the transmitter coordination system of brush tip location. The measurement was carried out during the calligraphic task for both novices and experts. The developed real-time eye-hand coordination system was used to measure eye movements and brush tip location during calligraphic tasks.

Examples of the positions of each brush tip stroke and eye- gaze are plotted shown in Fig.11 (skilled participant) and Fig. 12 (non-skilled participant) in Murata and Inoue ${ }^{9)}$. The brush tip and eye-gaze locations when writing the 5-th stroke of Kanji character "forever" are compared between a skilled and a non-skilled participant in Fig.13 in Murata and Inoue ${ }^{9)}$. As for the skilled participant, the brush tip location is distant from the eye-gaze location. As far as the novice is concerned, the brush tip exists at nearly the same location with the eye-gaze location. On the basis of the finding that the eye movement is preceding the brush tip for the skilled participant, it is possible that such a property might be one of the important skills necessary for acquiring skills in calligraphy.

In such a way, the proposed method for real-time measurement system of eye-hand coordination is promising for clarifying the process of skill acquisition in skilled movement such as calligraphy. Such a skilled element pointed out in the demonstrative experiment must be verified by a systematic experiment using the developed real-time measurement system of eye-hand coordination.

In addition to the difference mentioned above (Murata and Inoue $^{9)}$ ), time series of $x$-and $y$-directional difference between brush tip and eye-gaze point were obtained to analyze the difference between experts and novices in calligraphy. Moreover, the difference of calligraphy skill between skilled experts and novice (non-expert) was further analyzed using a pen pressure measurement system shown in Fig.1.

Fig. 2 shows the time series of $x$ - and $y$-directional difference between brush tip and eye-gaze point for a skilled participant. Fig. 3 shows the time series of $x$ - and $y$-directional difference between brush tip and eye-gaze point for a non-skilled participant. In Table 1, the results of paired t-test of maximum difference in $x$-axis and $y$-axis between skilled and non-skilled participants are shown for each of four kanji characters ( "forever" , "big" , "bright" , and "ups and downs" ). As well as the findings in Murata and Inoue ${ }^{9)}$ (see Figs.11-13), time series of $x$ - and $y$-directional difference between brush tip and eye-gaze point for skilled participants were larger than that of non-skilled participants (cf. Fig.2 and Fig.3). This tendency that the eye movement is preceding the brush tip for the skilled participant was further verified by a statistical analysis in Table 1.

Fig.4 and Fig.5 show examples of time series of writing pressure of each character for a skilled and non-skilled participant, respectively.

Fig. 6 shows examples of visual representation of change of writing pressure (skilled participant). Fig.7 shows examples of visual representation of change of writing pressure (skilled participant). Table 2 shows the results of paired t-test of pen pressure and S.D. of pen pressure between skilled and non- 


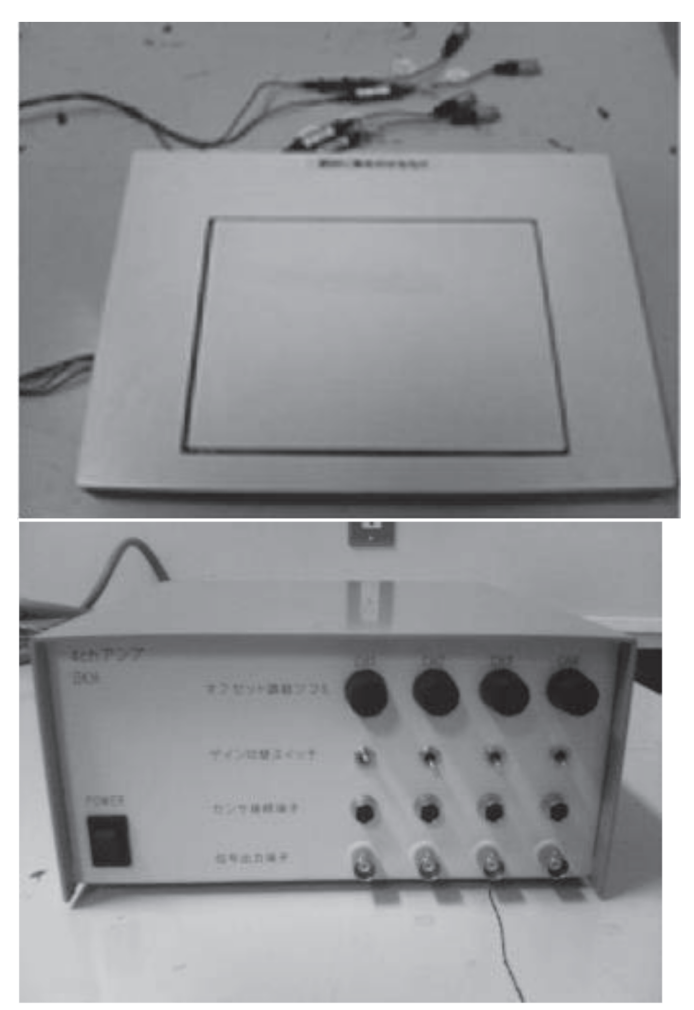

Fig.1 Outline of pen pressure measurement system (left: pen pressure measurement surface, right: 4-channel amplifier).
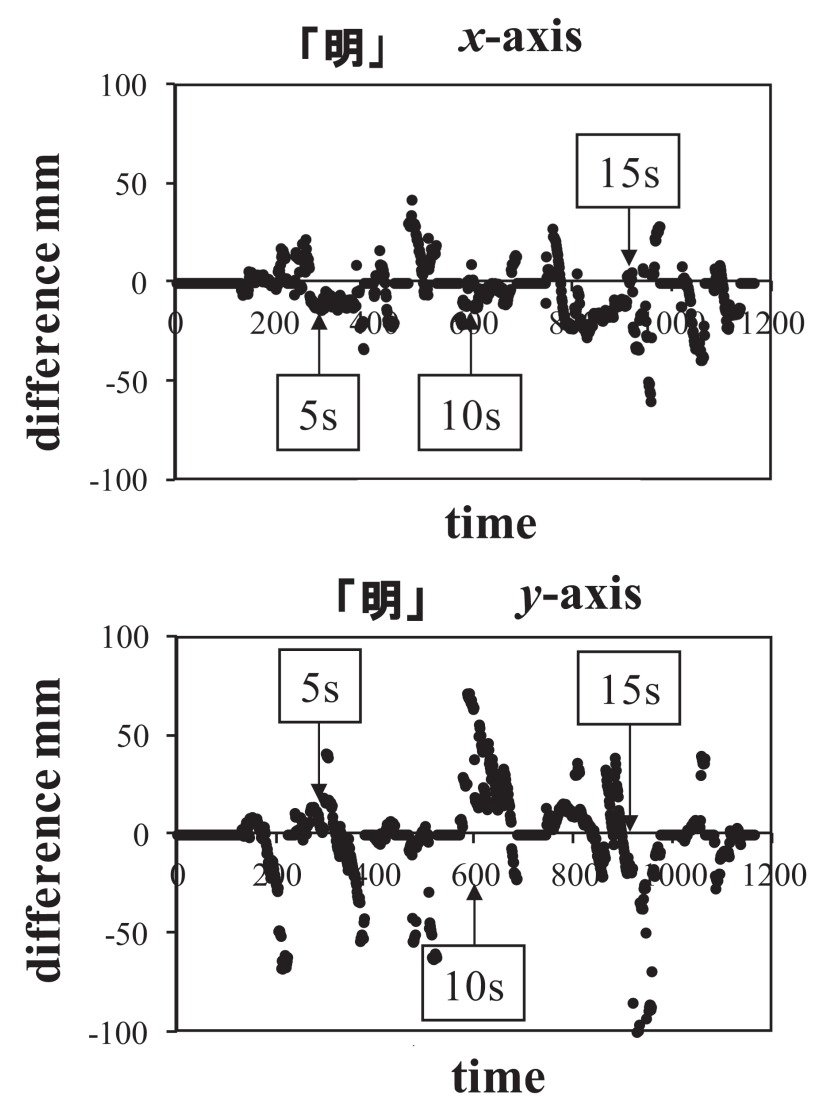

Fig.2 Time series of $x$ - and $y$-directional difference between brush tip and eye-gaze point (Examples of skilled participant).
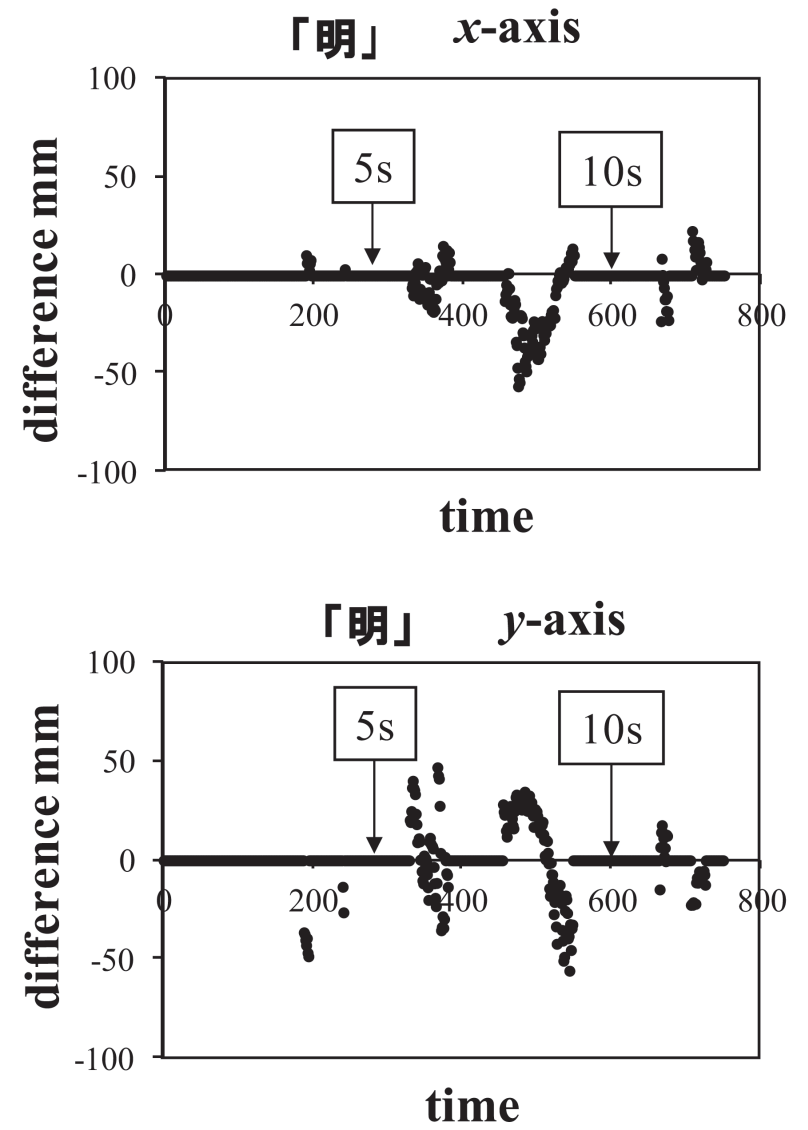

Fig. 3 Time series of $x$ - and $y$-directional difference between brush tip and eye-gaze point (Examples of non-skilled participant).

Table $1 t$-test of maximum difference in $x$-axis and $y$-axis between skilled and non-skilled

$*: p<0.05 * *: p<0.01$

\begin{tabular}{|c|c|c|c|}
\hline$x$-axis & skilled & non-skilled & $t$-statistics \\
\hline 永 (forever) & 62.71 & 23.55 & $t=5.489^{* *}$ \\
\hline 大 (big) & 47.08 & 33.59 & $t=3.064^{*}$ \\
\hline 明 (bright) & 47.20 & 26.60 & $t=4.340^{* *}$ \\
\hline 上下 (ups and downs) & 102.21 & 27.71 & $t=13.578^{* *}$ \\
\hline \multicolumn{4}{|c}{$*: p<0.05^{* *}: p<0.01$} \\
\hline
\end{tabular}

\begin{tabular}{|c|c|c|c|}
\hline$y$-axis & skilled & non-skilled & $t$-statistics \\
\hline 永 (forever) & 79.77 & 43.10 & $t=6.459^{* *}$ \\
\hline 大 (big) & 56.17 & 46.15 & $t=3.072^{*}$ \\
\hline 明 (bright) & 76.66 & 50.46 & $t=5.646^{* *}$ \\
\hline 上下 (ups and downs) & 70.80 & 30.15 & $t=6.781^{* *}$ \\
\hline
\end{tabular}

skilled participants.

The data indicate that the pen pressure of the expert participant was larger than that of the novice participant. Moreover, the pen pressure of the expert participant was more variable than that of the novice. This data indicate that the pen pressure of the expert is more intonated (sometimes strong and other times weak) than that of the novice. This must be the characteristic necessary for skilled calligraphy. 

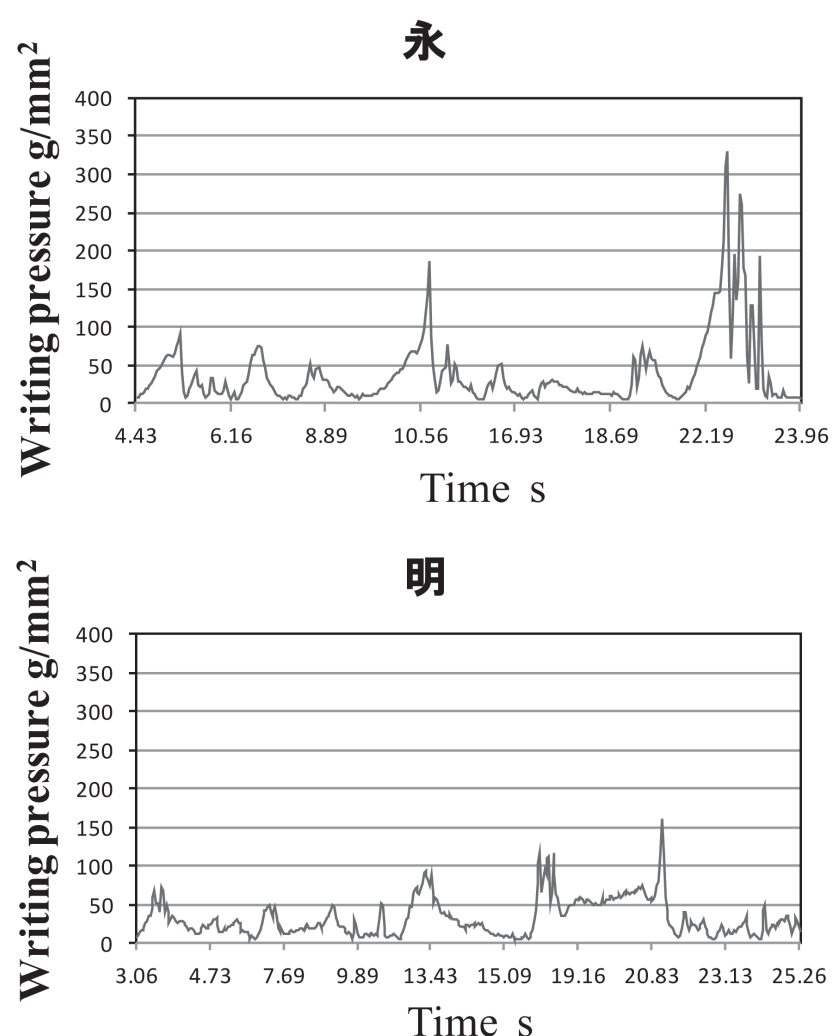

Fig.4 Examples of time series of writing pressure for each character (skilled participant).
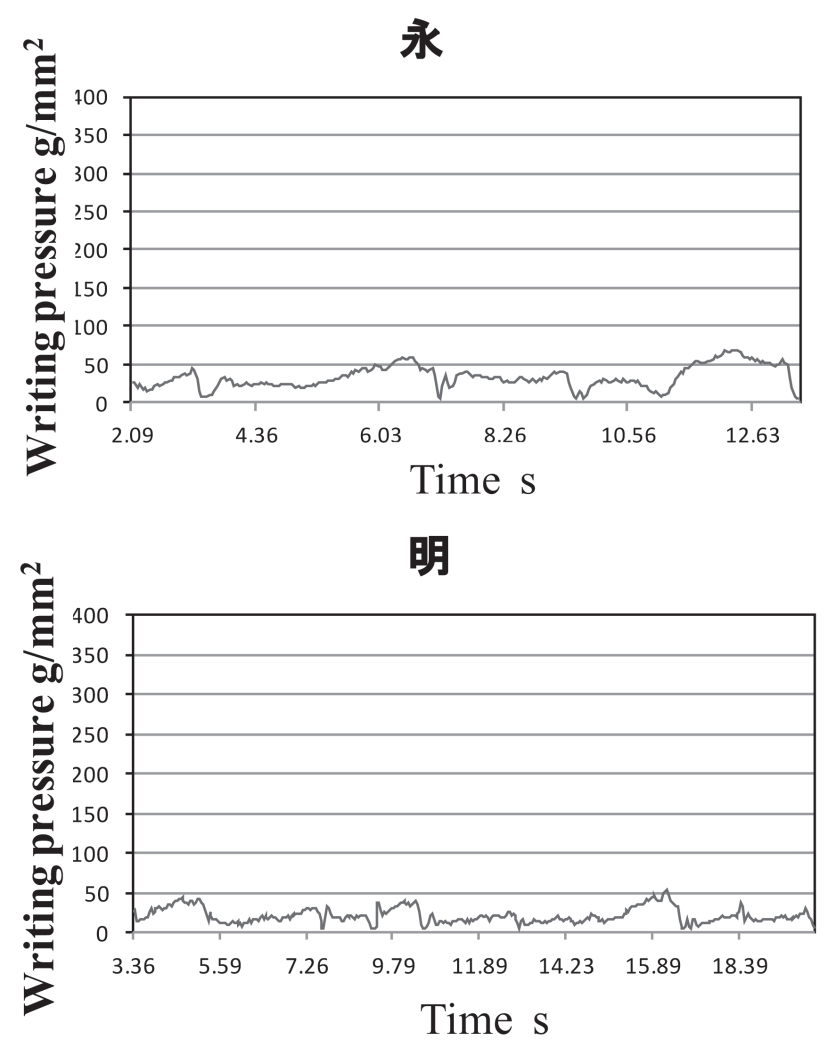

Fig.5 Examples of time series of pen pressure for each character (nonskilled participant).
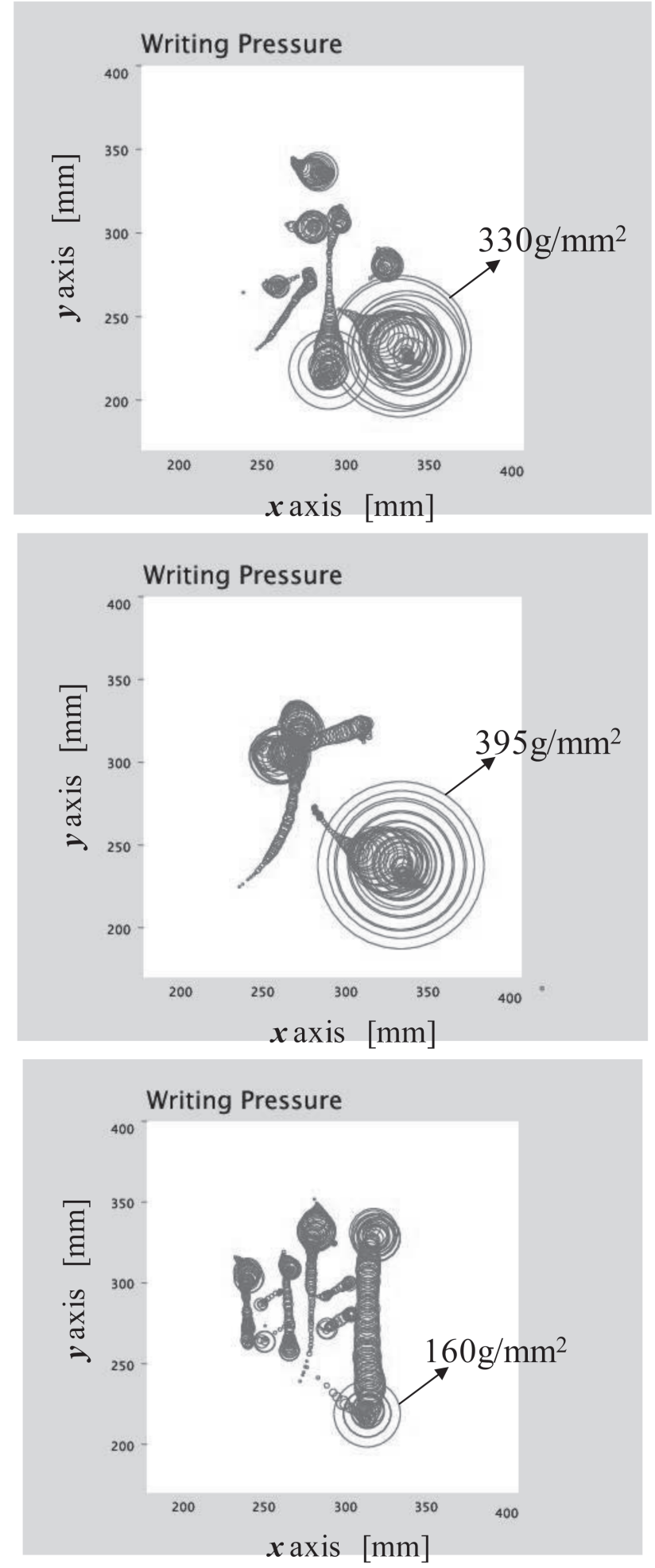

Fig.6 Examples of visual representation of change of writing pressure (skilled participant).

\section{Conclusions}

The aim of this stuffy was to explore eye-hand coordination skill necessary for enhancing performance from the perspective of simultaneous measurement of eye-gaze and pen tip locations 

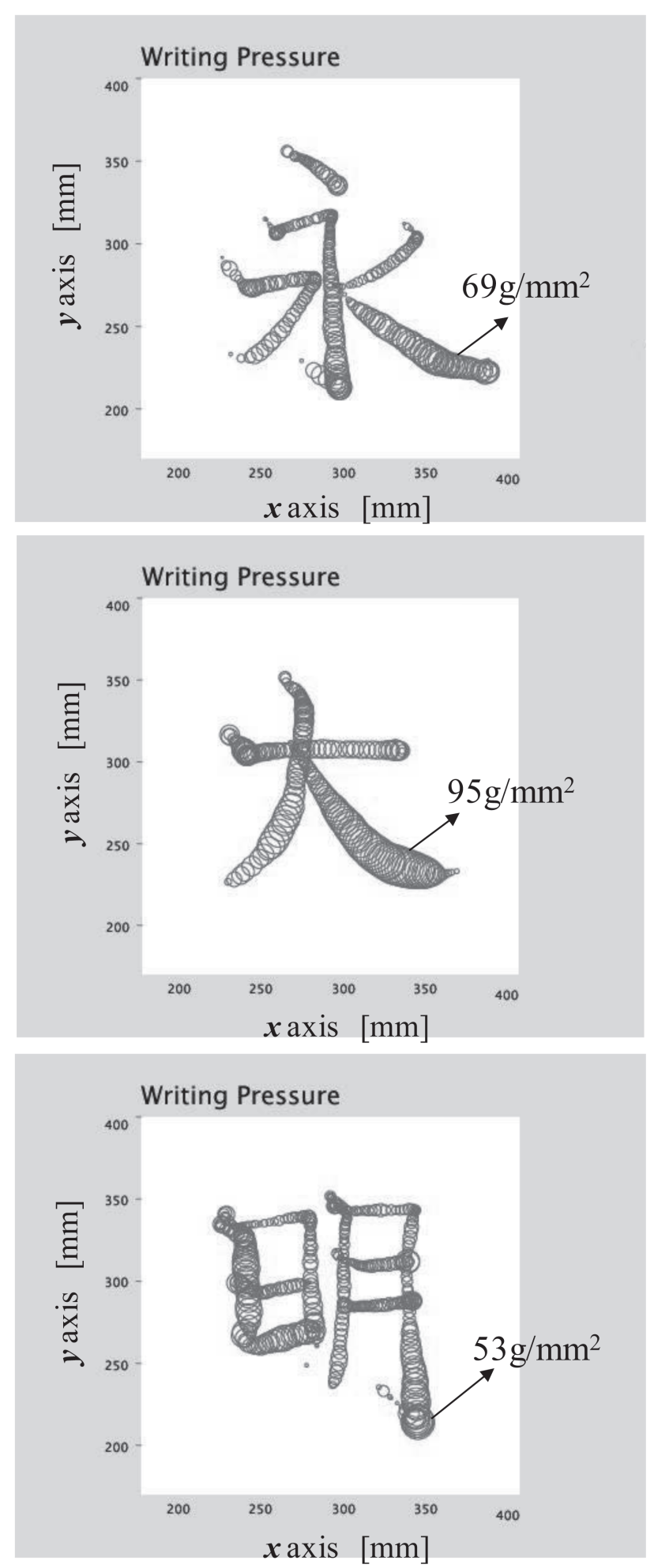

Fig.7 Examples of visual representation of change of writing pressure (non-skilled participant).

and writing (pen) pressure during a calligraphy task. The conclusions can be summarized as follows:

(1) Experts might be different from novices in the eye movement preceding brush tip, and such a property might be an important skilled element for acquiring a higher skill.
Table 2 t-test of writing pressure and S.D. of writing pressure between skilled and non-skilled participants.

$*: p<0.05 * *: p<0.01$

\begin{tabular}{|c|c|c|c|}
\hline Mean pen pressure & expert & novice & $t$-statistics \\
\hline 永 (forever) & 36.09 & 28.93 & $t=2.778^{*}$ \\
\hline 大 (big) & 56.05 & 39.21 & $t=2.144^{*}$ \\
\hline 明（bright） & 55.39 & 38.94 & $t=1.223$ \\
\hline \multicolumn{4}{|c|}{$*: p<0.05 * *: p<0.01$} \\
\hline Mean of S.D. of pen pressure & expert & novice & $t$-statistics \\
\hline 永 (forever) & 41.70 & 17.96 & $t=3.836^{* *}$ \\
\hline 大 (big) & 54.95 & 29.80 & $t=2.866^{*}$ \\
\hline 明（bright） & 49.04 & 18.76 & $t=2.533^{*}$ \\
\hline
\end{tabular}

(2) The $x$ - and y-directional difference between brush tip and eye-gaze point of the skilled participants was found to be larger than that of the novice participants.

(3) The fluctuation (intonation) of writing (pen) pressure of the skilled participant was larger than that of the novice participants.

The results should be further explored from the viewpoints of learning process (skill acquisition process). Future research should further verify the results (higher eye-hand coordination competence of calligraphy experts) in other fields. The simultaneous measurement system of eye-gaze and hand movement or the protocol to measure a variety of eye-hand coordination competence should widespread so that a lot of people can conveniently access and use such a device or protocol.

\section{References}

1) Johansson, R. S., Westling, G., Bäckström, A. and Flanagan, J. R.: Eye-hand coordination in object manipulation, Journal of Neuroscience, 21 (17), 6917-6932, (2001).

2) Bowman, M. C., Johannson, R. S. and Flanagan, J. R.: Eye-hand coordination in a sequential target contact task, Experimental Brain Research, 195 (2), 273-283, (2009).

3) Coen-Cagil, R., Coraggio, P., Napoletano, P., Schwartz, O., Ferraro, M. and Boccignone, G.: Visuomotor characterization of eye movements in a drawing task, Vision Research, 49 (8), 810-818, (2009).

4) Lazzari, S., Mottet, D. and Vercher, J. L.: Eye-hand coordination in rhythmical pointing, Journal of Motor Behavior, 41 (4), 294-304, (2009).

5) Crawford, J. D., Medendorp, W. P. and Marotta, J. J.: Spatial transformations for eye-hand coordination, Journal of Neurophysiology, 92 (1), 10-19, (2004).

6) Vickers, J.: Gaze control in basketball foul shooting, In 
Eye Movement Research: Mechanisms, Processes, and Applications, Eds. J. Findlay, R. Walker, and R. Kentridge, Elsevier, 527-541, (1995).

7) Murata, A. and Moriwaka, M.: Skill of eye-hand coordination in calligraphy -Difference of skill of eye-hand coordination between expert and novice-, Proceedings of 5th International Workshop on Computational Intelligence \& Applications, 316-319, (2009).

8) Sano, T. and Ukida, H.: Measurement of handwriting skills for Japanese calligraphy, Proceedings of Instrumentation and Measurement Technology Conference, 1112-1115, (2010).

9) Murata, A. and Inoue, K.: A method to measure eye-hand coordination for extracting skilled elements - Simultaneous measurement of eye-gaze and hand location -, Computer
Technology and Application, 5, 72-82, (2014).

\section{Atsuo MURATA}

Atsuo Murata received M.E and Ph.D. degree in industrial engineering in 1985 and 1987, respectively, from University of Osaka Prefecture. He was a Professor at Hiroshima City University from 1997 to 2006. From 2006, he is a Professor at Dept. of Intelligent Mechanical Systems, Okayama University. Professor Murata's current areas of research are mainly Cognitive Ergonomics, Accident Analysis and Safety Management, and Automotive Ergonomics. He is a member of Japan Ergonomics and Human Factors Society. 